\title{
Novel RET mutations in Hirschsprung's disease patients from the diverse South African population
}

\author{
Monique G Julies ${ }^{1}$, Sam W Moore ${ }^{2}$, Maritha J Kotze ${ }^{1}$ and Lana du Plessis ${ }^{*, 1}$ \\ ${ }^{1}$ Division of Human Genetics, Faculty of Medicine, University of Stellenbosch, Tygerberg, South Africa; ${ }^{2}$ Division of \\ Pediatric Surgery, Faculty of Medicine, University of Stellenbosch, Tygerberg, South Africa
}

Hirschsprung's disease (HSCR) is a common cause of intestinal obstruction in neonates with an incidence of one in $\mathbf{5 0 0 0}$ live births. The disease occurs due to the absence of parasympathetic neuronal ganglia in the hindgut, resulting in irregular or sustained contraction of the affected segment. DNA samples of 40 unrelated subjects with HSCR were subjected to mutation screening of the RET (REarranged during Transfection) protooncogene, the major susceptibility gene for HSCR. Five novel (V202M, E480K, IVS10-2A/G, D771N, IVS19-9C/ $\mathrm{T}$ ) and one previously described mutation (P973L) were identified. Only two of the mutation-positive patients (from different ethnic groups) displayed total colonic aganglionosis, and both were heterozygous for mutation D771N. The potential disease-causing mutations occurred in $20 \%$ of individuals, with more males (22.5\% representing seven of 31 males) affected than females (12.5\% representing one of eight females). This study represents the first comprehensive genetic analysis of this disease in the diverse South African population. European Journal of Human Genetics (2001) 9, 419-423.

Keywords: Hirschsprung's disease; RET proto-oncogene mutations; heteroduplex single strand conformational polymorphism (HEX-SSCP)

\section{Introduction}

Hirschsprung's disease (HSCR), or aganglionic megacolon, occurs due to the absence of parasympathetic neuronal ganglia in the hindgut and results in a malfunction of the affected segment. The condition therefore presents as a common cause of intestinal obstruction in neonates, with an incidence of one in $5000(0.02 \%)$ live births and an overall risk to siblings of $4 \% .^{1-3}$ Aganglionosis can involve the rectosigmoid area $(75 \%)$, transverse colon $(17 \%)$ or total colon $(8 \%)$ and may extend to the small bowel. Males are more likely to be affected than females (3.5-7.8-fold higher risk) and as the aganglionic segment becomes more extensive, the risk to siblings increases and the sex ratio decreases. ${ }^{4-6}$ A higher risk of disease occurrence has been reported in siblings of patients demonstrating an earlier age of onset.

\footnotetext{
*Correspondence: L du Plessis, Division of Human Genetics, Faculty of Medicine, PO Box 19063, University of Stellenbosch, 7505 Tygerberg, South Africa.

Tel: +27 21 9389212; Fax: +27 21 9317810; E-mail: Idp2@gerga.sun.ac.za Received 1 September 2000; revised 5 February 2001; accepted 6 March 2001
}

HSCR was believed to be a sex-modified multifactorial trait because males are more likely to be affected than females. ${ }^{1}$ Thereafter, inheritance was suggested to be multifactorial with the involvement of multiple genes and environmental factors in the development of the clinical phenotype. However, the occurrence of the disease in individuals displaying chromosomal abnormalities and sometimes multiple chromosome aberrations, suggested genetic heterogeneity. ${ }^{4}$ Chromosomal abnormalities such as Down's syndrome are frequently $(2-15 \%)$ associated with HSCR. ${ }^{7,8}$ The relatively constant association of Down's syndrome with HSCR disease suggests the involvement of a modifier gene on chromosome 21 in the pathogenesis of the disease. ${ }^{1,9}$

The first gene implicated in HSCR was the RET (REarranged during Transfection) proto-oncogene. This was demonstrated by the detection of an interstitial deletion of $10 \mathrm{q}$ occurring in a patient with long segment HSCR, as well as extensive segregation analysis of this area in large HSCR families demonstrating dominant inheritance. ${ }^{2,10,11} R E T$ involvement was substantiated by the identification of mutations associated with both familial and sporadic cases in $25 \%$ of all HSCR cases. ${ }^{10,12-16}$ RET mutations in sporadic 
HSCR occurs in approximately 40\% of patients with long segment disease and in $8 \%$ of patients with short segment disease. ${ }^{6}$ In both familial and sporadic cases of HSCR, RET mutations are not restricted to a particular area of the gene. In this study we performed mutation analysis of the RET gene in 40 unrelated HSCR patients from the diverse South African population.

\section{Materials and methods}

\section{Subjects}

In a retrospective study, colonic tissue samples from frozen tissue were obtained from 40 apparently unrelated HSCR patients and 30 control subjects (17 paraffin embedded and 13 frozen colonic tissue samples), including six patients with anorectal malformations. DNA samples, extracted from whole blood, of an additional 56 subjects were included as controls. Inclusion of HSCR patients were based on histopathological evaluation and confirmation of absence of ganglia in the biopsies. HSCR occurred sporadically in all of the patients, except in one individual with a family history of the disease. Total colonic aganglionosis was observed in eight of these patients and the remaining patients all had aganglionosis restricted to the rectosigmoid area.

The study population were from different ethnic groups of South Africa, including 51 subjects of the Coloured population (22 HSCR patients-55\%; 29 controls-34\%), 39 Blacks (nine HSCR patients-22.5\%; 30 controls-35\%;) and 33 Whites (eight HSCR patients-20\%, 25 controls-29\%). The ethnic origin of one HSCR patient (2.5\%) and two controls (2\%) were unknown. In this study the 'White' population was of European descent, mainly of British origin, 'Coloured' refers to an individual of mixed ancestry with descendants from San, Khoi, African Negro, Madagascar, Javanese and European origin; ${ }^{17}$ 'Black' refers to native Black populations of South Africa.

\section{DNA analysis}

DNA extraction was performed on the colonic tissue samples and whole blood using standard techniques. Polymerase chain reaction (PCR) amplification of the 21 exons of RET gene was performed using intronic primers. ${ }^{18}$ The PCR products were subjected to heteroduplex single-strand conformation polymorphism (HEX-SSCP) analysis, ${ }^{19}$ resolved in a polyacrylamide (PAA) gel supplemented with $7.5 \%$ urea (consisting of $4.5 \mathrm{~g}$ urea, $18 \mathrm{ml} 5 \times \mathrm{TBE}, 24 \mathrm{ml}$ $\mathrm{dH}_{2} \mathrm{O}, 18 \mathrm{ml}$ PAA (1\%C of a 40\% stock), $600 \mu \mathrm{l}$ APS (10\%) and $60 \mu \mathrm{l}$ TEMED) at $4{ }^{\circ} \mathrm{C}(350 \mathrm{~V})$ for $18 \mathrm{~h}$. The DNA fragments were also electrophoresed in a $10 \%$ PAA gel supplemented with $5 \%$ glycerol (consisting of $3 \mathrm{ml}$ glycerol, $6 \mathrm{ml} 5 \times \mathrm{TBE}$,


(10\%) and $80 \mu \mathrm{l}$ TEMED) at room temperature (300 V). The DNA fragments were stained in ethidium bromide and visualised by ultraviolet light transillumination. Semi-automated DNA sequencing (ABI 310 PRISM) was performed on
PCR products demonstrating mobility or conformational variants on the PAA gels.

\section{Statistical analysis}

Allele frequencies were determined and testing for significant association of alleles among patient and control groups were based on the chi-square $\left(\chi^{2}\right)$ and Fisher's exact tests. A $P$ value smaller than 0.05 was regarded as statistically significant.

\section{Results}

Mutation analysis of the RET proto-oncogene revealed six potential disease-related mutations in eight HSCR patients (Table 1). Nine polymorphims, of which five have been described previously ${ }^{14,18,20}$ were also detected (Table 2). HEXSSCP analysis showed no aberrant banding pattern in exons $1,4,5,8,9,10,12$ and 16 of the RET gene. Missense mutations and splice variants were identified in exons 3,7 , 13 and 17, and introns 10 and 19, respectively. The RET mutational spectrum defined in the South African population is illustrated in Figure 1.

All the patients were heterozygous for the respective mutations identified. The missense mutations $(4 / 6,66.6 \%)$ are not located to a specific hot-spot area of the RET protooncogene. None of the potential disease-associated mutations were detected in any of the 86 control individuals tested. Both mutations V202M and $\mathrm{E} 480 \mathrm{~K}$, detected in the extracellular domain of the RET proto-oncogene, were observed in men with aganglionosis restricted to the rectosigmoidal area. $\mathrm{E} 480 \mathrm{~K}$ was detected only in the Coloured population $(2 / 22,9 \%)$, however owing to the small numbers of the other two HSCR populations, the occurrence of this mutation in these populations cannot be excluded.

A mutation altering the splice site in intron 10 (IVS10-2A/ $\mathrm{G})$ was identified in a male with rectosigmoid aganglionosis. Mutation D771N was identified in two patients, from different ethnic backgrounds (patient 11, a Coloured male and patient 28, a White male), both with total colonic aganglionosis. A rare variant was identified in intron 16 (IVS16-38delG) in the same individual with missense mutation P973L. Only one patient with rectosigmoid aganglionosis presented with the IVS19-9C/T variant in intron 19.

Codon numbers were used for designation of the polymorphisms, because discrepancies were observed between the intronic sequences published by Ceccherini et $a l^{18}$ and Munnes et $a l^{21}$ (Blast \#AJ243297). Statistically significant differences in allele frequencies of the polymorphism identified in exon 15 of the RET gene was observed between the patient and control groups from the Black $(P<0.05)$ and White $(P<0.01)$ populations, respectively (Table 2$)$. The genotypes of the White and Coloured patients with mutation D771N were compatible with an identical chromosomal background for this mutation, whilst mutation E480K was associated with two different haplotypes. 
Table 1 Potential disease-causing mutations identified in the RET proto-oncogene

\begin{tabular}{|c|c|c|c|c|c|c|}
\hline Patient no. & Exon/Intron & Mutation & Nucleotide change & Effect on coding sequence & Gender/ethnic group & Extent of aganglionosis \\
\hline 18 & 7 & E480K & $\mathrm{GAA} \rightarrow \mathrm{AAA}$ & Missense & $M / C$ & $R / S$ \\
\hline 29 & 7 & E480K & $\mathrm{GAA} \rightarrow \mathrm{AAA}$ & Missense & $M / C$ & $R / S$ \\
\hline 11 & 13 & D771N & $\mathrm{GAC} \rightarrow \mathrm{AAC}$ & Missense & $M / C$ & TCA \\
\hline 28 & 13 & D771N & $\mathrm{GAC} \rightarrow \mathrm{AAC}$ & Missense & $\mathrm{M} / \mathrm{W}$ & TCA \\
\hline 36 & 17 & $\mathrm{P} 973 \mathrm{~L}^{\mathrm{a}}$ & CCA $\rightarrow$ ATA & Missense & $\mathrm{F} / \mathrm{C}$ & $\mathrm{R} / \mathrm{S}$ \\
\hline
\end{tabular}

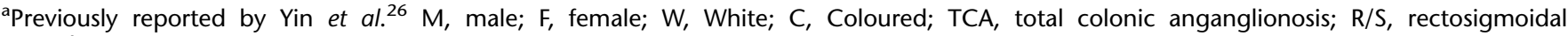
aganglionosis; IVS, intervening sequence.

Table 2 Polymorphisms identified in the RET proto-oncogene

\begin{tabular}{|c|c|c|c|c|c|c|c|c|}
\hline \multirow{4}{*}{$\frac{\text { Exon/Intron }}{2}$} & \multirow{3}{*}{$\frac{\text { Codon }}{\text { A45 }}$} & \multirow{3}{*}{$\begin{array}{l}\begin{array}{l}\text { Nucleotide } \\
\text { change }\end{array} \\
\mathrm{GCG} \rightarrow \mathrm{GCA}\end{array}$} & \multirow[b]{2}{*}{$B(n=18)$} & \multirow{3}{*}{$\frac{B C(n=60)}{0.94}$} & \multicolumn{2}{|c|}{ Allele frequency } & \multirow[b]{2}{*}{$C(n=44)$} & \multirow[b]{2}{*}{$C C(n=58)$} \\
\hline & & & & & $W(n=16)$ & $W C(n=50)$ & & \\
\hline & & & 0.95 & & 0.38 & 0.5 & 0.46 & 0.69 \\
\hline & & & 0.05 & 0.06 & 0.62 & 0.5 & 0.54 & 0.31 \\
\hline 6 & & & delG:0.0 & 0.0 & 0.06 & 0.0 & 0.11 & 0.10 \\
\hline \multirow[t]{2}{*}{7} & A432 & $\mathrm{GCG} \rightarrow \mathrm{GCA}$ & 1.0 & 1.0 & 1.0 & 0.94 & 0.85 & 0.88 \\
\hline & & & 0.0 & 0.0 & 0.0 & 0.06 & 0.15 & 0.12 \\
\hline \multirow[t]{2}{*}{13} & IVS13-29C/T & & 0.95 & 0.99 & 1.0 & 1.0 & 0.98 & 1.0 \\
\hline & & & 0.05 & 0.01 & 0.0 & 0.0 & 0.02 & 0.0 \\
\hline \multirow[t]{2}{*}{15} & 5904 & $\mathrm{TCC} \rightarrow \mathrm{TCG}$ & 0.50 & 0.75 & 0.82 & 0.94 & 0.57 & 0.59 \\
\hline & & & 0.50 & $0.25^{\mathrm{a}}$ & 0.18 & $0.06^{\mathrm{b}}$ & 0.43 & 0.41 \\
\hline \multirow[t]{2}{*}{16} & IVS16-38delG & & G: $\quad 1.0$ & 1.0 & 1.0 & 1.0 & 0.98 & 1.0 \\
\hline & & & delG:0.0 & 0.0 & 0.0 & 0.0 & 0.02 & 0.0 \\
\hline 18 & R982 & $\mathrm{CGC} \rightarrow \mathrm{CGT}$ & 1.0 & 0.92 & 0.88 & 0.9 & 0.91 & 0.97 \\
\hline
\end{tabular}

alack patients vs Black control group: $\chi^{2}=4.06 ; P>0.05{ }^{b}$ White patients vs White control group: $\chi^{2}=7.34 ; P>0.01$. B, Black patients; $B C$, Black control group; W, White patients; WC, White control group; C, Coloured patients; CC, Coloured control group.

\section{Discussion}

Mutation analysis of the RET gene in a series of 40 unrelated HSCR patients, revealed five novel and one previously described mutation in eight of 40 (20\%) individuals studied. None of the potential disease causing mutations were identified in a total of 86 control individuals tested. Notably, all the missense mutations identified in the study population involve amino acids that have remained evolutionarily conserved in human, mouse and rat. Nine polymorphisms, of which three were novel, were also detected. Similar to previous studies, the mutations identified in $R E T$ are scattered throughout the gene. The percentage of individuals presenting with RET mutations in this study (20\%) is consistent with previous findings of approximately $25 \%$ of HSCR cases caused by RET mutations. ${ }^{12,14}$

The majority of mutations $(66.7 \%)$ were detected in the Coloured population which constitutes the largest study group, with mutation E480K in exon 7 occurring in two of 22 Coloured patients $(9 \%)$. The remainder of the mutations identified (33.3\%), occurred in White patients. No mutations were identified in the Black population. Two mutations were identified in the extracellular domain of the gene, encompassing exon 3 (V202M) and exon 7 (E480K). Exons 3 and 7 partly encode the cadherin-like domain and the cysteine-rich area, respectively. ${ }^{22,23}$ In this study, no functional analysis was performed on the mutations identified in the RET gene, but the likelihood exist that mutations in this area may cause RET loss-of-function by a dominant-negative mechanism. ${ }^{24}$

A splice variant (IVS10-2A/G) was identified in intron 10 in an individual with aganglionosis in the rectosigmoid area. A sequence variant identified in the pyrimidine tract of the splice acceptor of intron 19 (IVS19-9C/T), with a pyrimidine to a pyrimidine change is probably not pathologic. ${ }^{25}$ Mutations in exon 13 (D771N) and exon 17 (P973L) involve 

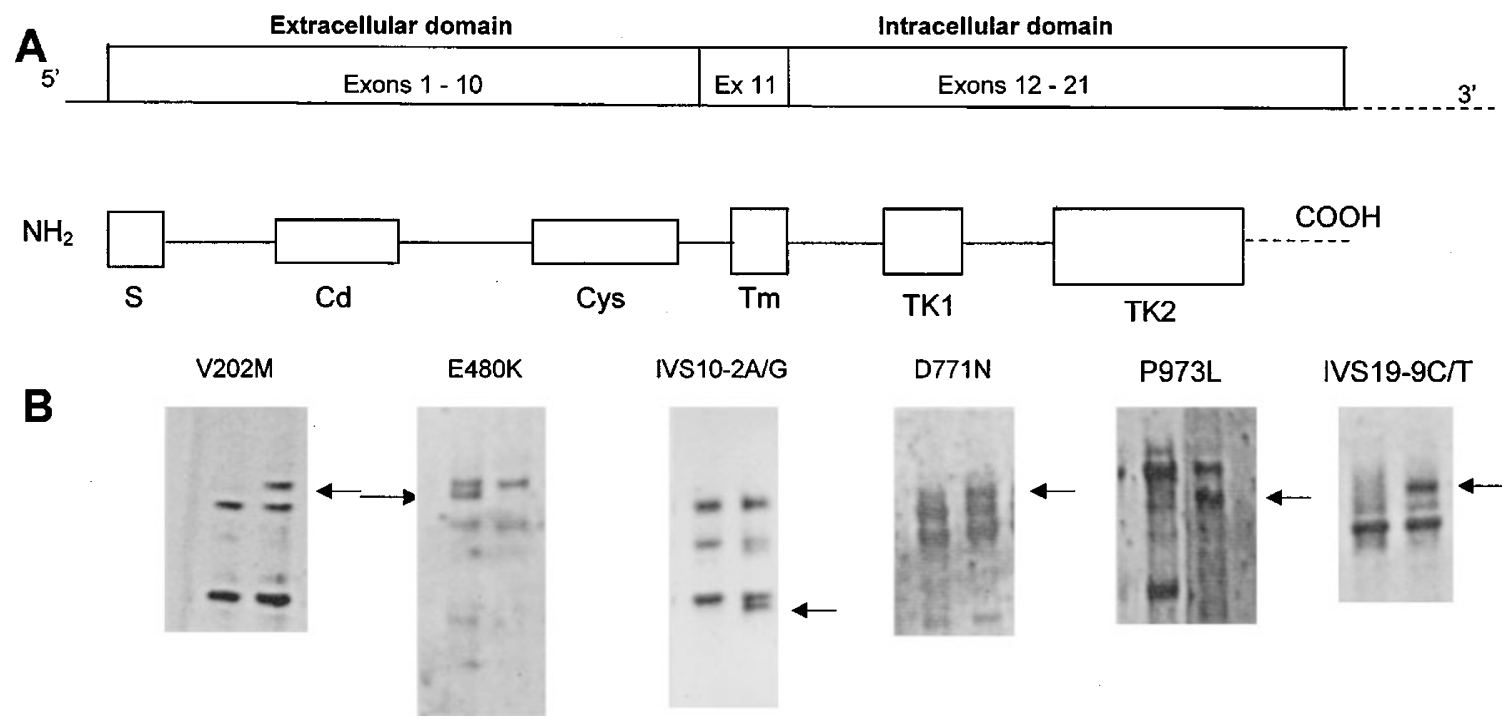

Figure 1 Schematic representation of genomic organisation of the RET proto-oncogene in relation to three distinct functional domains of the protein. Mutations identified in the South African population are shown. A, Exons encoding for specific domains of the RET protein. B, Ethidium bromide stained gel images of PCR-SSCP banding patterns of mutations in $12 \%$ polyacrylamide gels supplemented with 7.5\% urea. Arrows indicate abnormal bands. S, signal peptide; Cd, cadherin-like sequence; Cys, cysteine-rich region; Tm, transmembrane domain; TK1 and TK2, split tyrosine kinase regions 1 and 2.

the intracellular domain, the most highly conserved area of the gene. The mutation in exon 17 (P973L) was previously described by Yin et $a^{26}$ who speculated that this mutation might inactivate $R E T$ gene function. The variant identified in intron 16 (IVS16-38delG) in one of the HSCR patients with mutation P973L, might contribute to disease modification and only by functional analysis will the role of this rare association be determined.

Although the numbers were small for statistical analysis, a varying trend was observed upon comparison of the exon 15 polymorphism of the RET gene in both the Black and White patients $v s$ the respective control groups. Comparison of allelic frequencies of variant S904 in the total HSCR patient group with the total control group, demonstrated a statistically significant difference $\left(P<0.05, \chi^{2}=5.7\right)$ which is consistent with previous findings of over-representation of the wild type allele in the HSCR population. ${ }^{27,28}$ The observed trend of polymorphic allelic frequency variation in the HSCR and the mainly blood-derived control group, could indicate a higher susceptibility of specific tissues to mutational change. Of interest in a recent study conducted in patients presenting with HSCR and Down's syndrome, different RET polymorphisms were identified in the same individual when areas of normal ganglionic development were compared to regions of aganglionosis ( $\mathrm{L}$ du Plessis, unpublished data). Four of the nine variants identified in this study, IVS6+56delG, A432, IVS13-29C/T and IVS16-38delG, may possibly be low penetrant susceptibility alleles. In light of the role that RET plays in the cellular signalling pathway between different cells, these findings warrant further investigation as recent evidence indicates that silent mutations may influence $R E T$ transcription and lead to altered protein levels causing functional changes in RET itself or through interaction with other closely linked mutated genes. ${ }^{27,28}$ The likelihood that variant $\$ 904$ may be in linkage disequilibrium with other disease-causing mutation(s) in the gene should also be considered, particularly in the Black patient group where no mutations could be found. Failure to detect RET mutations in Blacks is probably a consequence of the heterogeneous nature of HSCR implying a different genetic basis in this group, but limitations imposed by the HEX-SSCP screening method ${ }^{19}$ (mutation detection efficiency $\sim 90 \%$ ) used may also account for the apparent absence of RET mutations in the Blacks with HSCR.

Other genes involved in HSCR includes the endothelin- $B$ receptor (EDNRB) gene and its ligand the endothelin-3 (EDN3) gene, ${ }^{9,29}$ the glial cell line-derived neurotrophic factor (GDNF) gene, ${ }^{30,31}$ endothelin-converting enzyme 1 (ECE1), ${ }^{32}$ the sex dependent $Y$ factor-like homeobox 10 (SOX10) gene $^{33}$ and neurturin (NTN). ${ }^{34}$ Studies are currently underway to screen the $E D N R B$ and $E D N 3$ genes for sequence variation and altered expression in our study population. Future genetic analysis of HSCR in particularly the Coloured population of South Africa, recently defined as a potential candidate of admixture linkage disequilibrium approaches to identify causative genes/mutations, ${ }^{35}$ may contribute significantly to a complete understanding of the complex interaction of genes involved in neural crest derived pathologies.

This study represents the first genetic analysis of HSCR in the diverse South African population, and highlights the 
importance of the RET proto-oncogene in neurocristopathologic disease. Although no direct evidence concerning the causative nature of the novel RET mutations is provided, either through loss of activity, enhanced activity or altered specificity of the protein, several lines of evidence support their significance in HSCR. Detection of identical mutations in patients from different ethnic groups (D771N) or associated with possible distinct haplotypes in the same population group (E408K), whilst absent in the control groups, signifies the relative importance of the relevant protein domains in HSCR.

\section{Acknowledgements}

This work was financially supported by the Harry and Doris Crossley Foundation. MG Julies received a student bursary from the South African Medical Research Council.

\section{References}

1 Passarge E: The genetics of Hirschsprung's disease: evidence for heterogeneous etiology and a study of sixty-three families. New Eng J Med 1967; 276: 138-143.

2 Angrist M, Kauffman E, Slaugenhaupt $S$ et al: A gene for Hirschsprung disease (megacolon) in the pericentromeric region of human chromosome 10. Nat Genet 1993; 4: 351-356.

3 Lyonnet S, Bolino A, Pelet A et al: A gene for Hirschsprung's disease maps to the proximal long arm of chromosome 10. Nat Genet 1993; 4: 346-350.

4 Badner J, Sieber W, Garver K, Chakravarti A: A genetic study of Hirschsprung's disease. Am J Hum Genet 1990; 6: 568-580.

5 Moore SW, Rode H, Millar AJ, Albertyn R, Cywes S: Familial aspects of Hirschsprung's disease. Eur J Pediatr Surg 1991; 1: 97 101

6 Puri P: Hirschsprung's disease; in Oldham TO, Colombani PM, Foglia RP (eds): Surgery of infants and children: Scientific principles and practice. New York, Lippencott-Raven, 1997, chap 80, pp $1277-1299$.

7 Polly T, Coran A: Hirschsprung's disease in the newborn. Pediatr Surg Int 1993; 1: 80-83.

8 Puri P: Hirschsprung's disease: Clinical and experimental observations. World J Surg 1993; 17: 374-384.

9 Puffenberger E, Kauffman E, Bolk S et al: Identity-by-descent and association mapping of a recessive gene for Hirschsprung disease on human chromosome 13q22. Hum Mol Genet 1994; 3: $1217-1225$.

10 Angrist M, Bolk S, Thiel B et al: Mutation analysis of the RET receptor tyrosine kinase in Hirschsprung disease. Hum Mol Genet 1995; 4: 821-830.

11 Luo Y, Ceccherini I, Pasini B et al: Close linkage with the RET proto-oncogene and boundaries of deletion mutations in autosomal dominant Hirschsprung disease. Hum Mol Genet 1993; 2: $1803-1808$.

12 Edery P, Lyonnet S, Mulligan L et al: Mutations of the RET protooncogene in Hirschsprung's disease. Nature 1994; 367: 378 - 380.

13 Luo Y, Barone V, Seri M et al: Heterogeneity and low detection rate of RET mutations in Hirschsprung disease. Eur J Hum Genet 1994; 2: $272-280$.

14 Romeo G, Ronchetto P, Luo Y et al: Point mutations affecting the tyrosine kinase domain of the RET proto-oncogene in Hirschsprung's disease. Nature 1994; 367: 378 - 380.

15 Attie T, Pelet A, Edery P et al: Diversity of RET proto-oncogene mutations in familial and sporadic Hirschsprung disease. Hum Mol Genet 1995; 4: 1381-1386.
16 Seri M, Luo Y, Barone A et al: Frequency of RET mutations in long and short-segment Hirschsprung disease. Hum Mut 1997; 9: $243-249$.

17 Nurse GT, Weiner JS, Jenkins T (eds): The growth of hybrid communities; in The Peoples of Southern Africa and their Affinities. Oxford, Clarendon Press, 1985, pp 218-224.

18 Ceccherini I, Hofstra R, Luo Y et al: DNA polymorphisms and conditions for SSCP analysis of the 20 exons of the RET protooncogene. Oncogene 1994; 9: 3025-3029.

19 Kotze M, Theart L, Callis M, Peeters A, Thiart R, Langenhoven E: Nonradioactive multiplex PCR screening strategy for the simultaneous detection of multiple low-density lipoprotein receptor gene mutations. PCR Methods Applic 1995; 4: 352-356.

20 Edery P, Attie T, Mulligan L et al: A novel polymorphism in the coding sequence of the human RET proto-oncogene. Hum Genet 1994; 94: 579-580.

21 Munnes M, Fanaei S, Schmitz B, Muiznieks I, Holschneider AM, Doerfler W: Familial form of hirschsprung disease: nucleotide sequence studies reveal point mutations in the RET protooncogene in two of six families but not in other candidate genes. Am J Med Genet 2000; 4;94:19-27.

22 Takeichi M: Cadherin cell adhesion receptors as a morphogenetic regulator. Science 1991; 251: 1451-1455.

23 Overduin M, Harvey T, Bagby S et al: Solution structure of the epithelial cadherin domain responsible for selective cell adhesion. Science 1995; 267: 386-389.

24 Cosma M, Cardone M, Carlomagno F, Colantuoni V: Mutations in the extracellular domain cause RET loss of function by a dominant negative mechanism. Mol Cell Biol 1998; 18: 3321 3329.

25 Krawczak M, Reiss J, Cooper DN: The mutational spectrum of single base-pair substitutions in mRNA splice junctions of human genes: causes and consequences. Hum Genet 1992; 90: $41-54$.

26 Yin L, Barone V, Seri M et al: Heterogeneity and low detection rate of RET mutations in Hirschsprung disease. Eur J Hum Genet 1994; 2: 272-280.

27 Borrego S, Sáez M, Ruiz A et al: Specific polymorphisms in the RET proto-oncogene are over-represented in patients with Hirschsprung's disease and may represent loci modifying phenotypic expression. J Med Genet 1999; 36: 771-774.

28 Fitze G, Schreiber M, Kuhlisch E, Schackert H, Roesner D: Association of RET proto-oncogene codon 45 polymorphism with Hirschsprung disease. Am J Hum Genet 1999; 65: 1469-1473.

29 Bolk S, Angrist M, Xie J et al: Endothelin-3 frameshift mutation in congenital central hypoventilation syndrome. Nat Genet 1996; 13: 395 - 396.

30 Jing S, Wen D, Yu Y et al: GDNF-induced activation of the RET protein tyrosine kinase is mediated by GDNFR-alpha, a novel receptor for GDNF. Cell 1996; 85: 1113-1124.

31 Treanor J, Goodman L, de Sauvage F et al: Characterisation of a multicomponent receptor for GDNF. Nature 1996; 382: 80-83.

32 Hofstra R, Valdenaire O, Arch E et al: A loss-of-function mutation in the endothelin-converting enzyme 1 (ECE-1) associated with Hirschsprung disease, cardiac defects, and autonomic dysfunction. (Letter) Am J Hum Genet 1999; 64: 304-308.

33 Pingault V, Puliti A, Prehu M-O, Samadi A, Bondurand N, Goossens M: Human homology and candidate genes for the dominant megacolon locus, a mouse model of Hirschsprung disease. Genomics 1997; 39: 86-89.

34 Doray B, Salomon R, Amiel J et al: Mutation of the RET ligand, neurturin, supports multigenic inheritance in Hirschsprung disease. Hum Mol Genet 1998; 7: 1449-1452.

35 Loubser O, Marais AD, Kotze MJ et al: Founder mutations in the LDL receptor gene contribute significantly to the familial hypercholerolemia phenotype in the indigenous South African population of mixed ancestry. Clin Genet 1999; 55: 340-345. 